\title{
Información social en la era de la democracia electrónica
}

\section{Silvia Pellegrini}

Periodista, Decana de la Facultad de Letras y Periodismo de la

Universidad Católica de Chile [spellegr@puc.cl]

La tecnología altera los conceptos de soberanía, sociedad y gobierno, y promueve una restricción de los tiempos y funciones de la burocracia. Pero a la vez, amplía los mecanismos de participación, lo que ha permitido que aparezca una "democracia electrónica". La interconectividad -sostiene la autora-incide en la generación de problemas en la esfera de la comprobación de la veracidad y confiabilidad de los datos, y en toda la posibilidad periodística de ofrecer "pautas confiables de navegación" a los usuarios directos.
I concepto base de democracia elec- trónica, que consiste en un ciudadano interactuando con los organismos de gobierno, informándose, votando y haciendo funciones burocráticas a través del computador, no parece de ciencia ficción, por distante que esté de algunas realidades sociales.

Pero esa perspectiva implica, sin duda, modificaciones importantes en el sistema democrático, tal como lo concebimos durante largas décadas del siglo XX.

La democracia es hoy el modo de organización política. Ampliamente difundido en el mundo, lo que se discute ya no es sobre lo adecuado o inadecuado de esa forma de gobierno, sino sobre la calidad de cada democracia particular.

Pero, aún sin poner en tela de juicio la validez del sistema, nunca ha habido tanto desprestigio del ámbito político mismo, ni tan abierto descontento de los gobernados con sus gobernantes, como en esta década.

Parte de esa valoración negativa de lo político podría deberse a la incorporación de vastas mayorías sociales a la participación social, al consumo y a la información. La política ha perdido no sólo prestigio, 
sino también interés frente a esas dos últimas áreas que aparecen como los principales conceptos emergentes. Pero el desarrollo tecnológico influye también, en la medida en que pone en evidencia algunas de sus deficiencias y le plantea nuevos desafíos.

La tecnología y sus sistemas abiertos de generación e intercambio de información han hecho cambiar, por ejemplo, los márgenes de la idea de soberanía, los alcances del concepto de sociedad y, por tanto, de las actuales formas usadas por la democracia para gobernarla.

Además, en la medida en que ha aumentado vertiginosamente la velocidad de las transmisiones informativas, se han producido cambios substanciales en el tiempo de conocimiento de los hechos, hasta prácticamente acercarse a lo que se ha llamado "tiempo real", es decir, a la simultaneidad entre el hecho y la información. La tecnología ha llenado también brechas espaciales, hasta hacer cercanos lugares muy remotos.

Por contrapartida, los tiempos de las decisiones burocráticas y de gobierno continúan siendo los mismos, y ello contribuye a la crítica sobre la efectividad de la democracia. Esa situación obliga a las instituciones democráticas a intentar alguna suerte de presencia de sus decisiones dentro de los tiempos informativos, generando a veces, pseudo-informaciones o informaciones insubstanciales, para responder a los requerimientos de los medios. Lo que no contribuye a prestigiarlas.

El proceso someramente descrito tiene, en realidad, variadas formas de influencia de la tecnología electrónica en el proceso democrático, que pueden resumirse en tres grandes áreas: ciertas modificaciones en la forma de ejercer el gobierno; una restricción de los tiempos y funciones de la burocracia y una ampliación de los mecanismos de participación.

\section{TECNOLOGÍA COMO MODIFICACIÓN DE LAS FORMAS DE GOBIERNO.}

En el contexto de la democracia electrónica, el Estado se ve forzado, o impedido al menos, a un compromiso con la permanente expansión de la información: para incorporarla como una de sus políticas de desarrollo y para responder frente a sus gobernados con información fidedigna, en lo que se ha llamado "la transparencia administrativan.

Incorporar la tecnología electrónica como una de sus políticas significa contribuir a la proliferación de una estructura tecnológica, tanto en lo que se refiere a la incorporación de hard y software en su ámbito de acción, como a la inversión en investigación sobre nuevas aplicaciones tecnológico-informativas.

Esto es especialmente válido en los países en vía de desarrollo, donde, además, es necesario propiciar que el uso de la tecnología abarque a los más amplios sectores sociales. Esa proliferación de la estructura tecnológica incluye también, en América Latina algo que está ampliamente logrado en los países del primer mundo: la capacitación del usuario en el acceso al pensamiento y uso computacional. Ambos elementos son la base para lograr la llamada "interconectividad electrónica" de todos los miembros de la sociedad.

En esa perspectiva, el Vicepresidente norteamericano $\mathrm{Al}$ Gore, en su discurso a la Unión Internacional de Telecomunicaciones en Buenos Aires, planteaba la posibilidad de crear la Infraestructura Mundial de la Información (IMI), con redes locales interconectadas a otras internacionales de mayor cobertura.

Un segundo aspecto de cambio de las formas de gobierno, en la era de la democracia electrónica, es el compromiso que se le plantea de aportar a sus gobernados la mayor riqueza informativa disponible. En especial de hacer conocido todo aquel flujo de datos que deriva de sus propias áreas de acción y depende de sus propias fuentes. Ese compromiso requiere, también, ofrecer un acceso fácil, organizado y veraz a las decisiones en la esfera gubernamental (resumido en el concepto de "organización de la transparenciaw) y una garantía de la más amplia libertad de expresión, para contribuir a la difusión de toda esa información.

Una pauta-guía en World Wide Web, por ejemplo, podría aportar un excelente

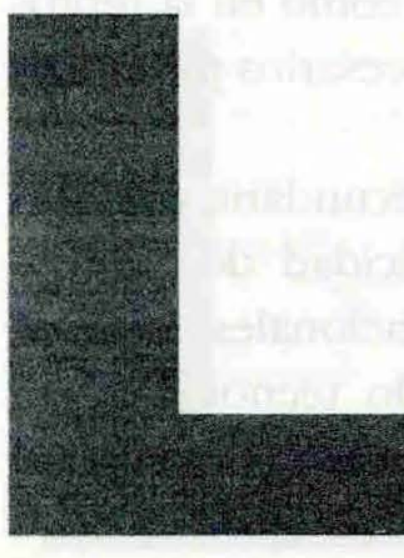

\section{a tecnología y sus sistemas abiertos de generación e intercambio de información han hecho cambiar los márgenes de la idea de soberanía, los alcances del concepto de sociedad.}




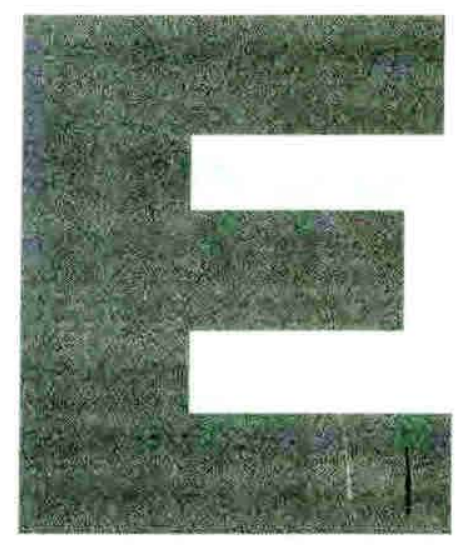

\section{I logro de la transparencia administrativa es una función que excede a los medios de comunicación y compete, de hecho, a toda la sociedad. En América} Latina implica romper la llamada «cultura del secreto».

marco para el acceso a esta información organizada. Esa guía debería ser un componente básico de esta red.

Pero hasta hoy, son otras las alternativas que han usado los organismos gubernamentales: la primera es incluir en ella, tanto información base o publicitaria sobre los participantes u organización del organismo respectivo, como incorporar documentos informativos, reglamentos y actas de decisiones de esos organismos y sus interrelaciones burocráticas. Es el conjunto de estas tres vertientes de información lo que constituye la verdadera transparencia administrativa.

La democracia norteamericana, por ejemplo, tiene ya más de cien mil documentos disponibles en Internet, de acuerdo con datos suministrados por Michael Nelson, a cargo de la información tecnológica de la Casa Blanca. Chile, en cambio, está iniciando su incorporación plena a esta red, ya que si bien hay numerosísimos usuarios personales, las home page gubernamentales recién comienzan a aparecer, y la información documental es aún insuficiente.

Otra forma de aumento de la transparencia administrativa es lo que implica la ley norteamericana llamada "Government in the Sunshine Act" (Gobierno a la luz del soln), que lleva ese concepto hasta hacer públicas algunas reuniones de organismos de la Administración.

Curiosamente, ese tipo de medidas pue- de producir exactamente el efecto contrario del buscado: como muchas cosas administrativas no pueden llevarse, así como así, a la luz pública, surge una forma de realidad virtual informativa. Es decir, la posibilidad de que estas reuniones sean solamente la apariencia y no la instancia efectiva de toma de decisiones, y que, de hecho, se genere un doble estándar en ese proceso, que permita mantener la reserva, ya sea temporal o parcial de ciertas situaciones.

El logro de la transparencia administrativa es una función que excede a los medios de comunicación y compete, de hecho, a toda la sociedad. En América Latina implica romper la llamada "cultura del secreto", donde en la administración pública, de modo regular, sólo se habla de aquéllo que explícitamente se ha establecido como público, silenciando vastas áreas que no tienen relación alguna con lo que debiera ser secreto social (un concepto referido exclusivamente a hechos que ponen al Estado-nación en peligro).

Adicionalmente, esa cultura del secreto afecta también a las empresas privadas. Éstas, por su condición, no tienen la misma obligación de informar que el Estado, pero extienden el velo de lo secreto mucho más allá de aquéllo que pudiera considerarse como reserva industrial, y tienden, asimismo, a recelar de cualquier información que no sea publicitaria, con respecto de su función.

\section{TECNOLOGIAA RESTRICTIVA DEL TIEMPO Y LAS FUNCIONES BUROCRÁTICAS}

Otro ángulo de acción de la democracia electrónica es la restricción del tiempo y de las funciones burocráticas. Entre sus alternativas, está la posibilidad de acceder a una serie de documentos, certificados y otros similares en el momento mismo de solicitarlos, votar desde el computador a través de la identificación de la voz o de la huella digital puesta en la pantalla, o hacer la presentación de cualquier solicitud desde la casa, por ejemplo. Una interconexión en red de los diversos servicios públicos, reduciría, incluso, la necesidad de la existencia de determinados certificados, con la consiguiente disminución de la cantidad de público que acudiera a las diversas oficinas.

Otra forma de reducción de tiempos y costos por las redes informativas estriba en la generación de amplias bibliotecas y videotecas accesibles por computación. Paralelamente al efecto en la difusión masiva del conocimiento, esta posibilidad reduce costos de personal y espacio tanto en la mantención de ellas, como en la reproducción de los datos necesarios para cualquier uso.

Una consecuencia secundaria y no deseable de esta multiplicidad de interconexión de datos computacionales es que se convierta este desarrollo tecnológico en una forma opresora de las personas, al poner a disposición de cualquiera, todos 
sus datos privados. También plantea peligros a la propiedad intelectual por la reproducción de especies intelectuales o artísticas. Estas posibilidades han dado origen a diversas legislaciones sobre el uso de bases de datos y material computacional. La ley federal estadounidense sobre el derecho a la confidencialidad (1974), por ejemplo, plantea que toda persona tiene derecho a que los datos de sus expedientes sean exactos y no estén sometidos a un uso indebido o indiscriminado.

\section{TECNOLOGIAA PARA LA PARTICIPACIÓN}

Una perspectiva complementaria a los cambios que introduce la tecnología en las formas de gobierno y en la burocracia es mirarla como promotora de la participación informada del público en las decisiones del poder administrativo, sea por la vía de una mayor información política, por la posibilidad de ampliación de los actos de tipo plebiscitario, por un adecuado uso del feedback, o por una mayor velocidad en las encuestas y resultados eleccionarios.

Este ángulo apunta a ampliar esa característica esencial del régimen democrático que es el delicado equilibrio entre libertad e igualdad de las personas, a través de la calidad y cantidad de información que éstas reciban para abordar las decisiones democráticas que les competen.

En el sentido participativo del uso tecnológico, se enmarcan las leyes que impul- san a los medios a ofrecer igualdad de tiempo informativo a los candidatos electorales, por ejemplo. Eso se consigue en Chile a través de la ley de elecciones, que establece tiempos iguales a los candidatos presidenciales y proporcionales a la representación parlamentaria de los partidos en las elecciones de congresales. La ley impone la gratuidad de esta "franja electoral" para las estaciones televisivas de libre recepción lo que, por otra parte, ha generado serios reclamos de las afectadas.

Un concepto similar existe en EE.UU., donde la ley de comunicaciones, ya en 1934, estipulaba que si una estación radiodifusora ofrece tiempo a un candidato debería ofrecerle igual tiempo a los otros. (Diversas interpretaciones posteriores de esa ley han hecho que esta igualdad de tiempo no se aplique a los noticieros, pero sí a la venta de tiempo publicitario, que obliga a que si le venden a uno, el medio ha de venderle también a los demás.)

Una segunda forma de usar la electrónica para la participación es la mayor frecuencia con que se puede recurrir a actos de tipo plebiscitario, vía Internet, por ejemplo, para obtener opiniones directas del público.

Un artículo escrito por la revista The Economist del 17 de junio de 1995, habla de la "versión rosa" y la "versión negra" de esta participación cuasi plebiscitaria. Una es la de una mayor instancia real de participación, que Heidi y Alvin Toffler en su libro
Las guerras del futuro han llamado "la democracia semi-directan; la segunda, se refiere a la posibilidad de que cualquier persona emita por Internet cualquier idea y miles de usuarios la voten a favor o en contra, lo que es una peligrosa versión de democracia directa.

Existe, por último, una tercera forma participativa electrónica que es la del feed back desde las personas hacia el gobierno. Es la forma de uso bidireccional de la red, como medio de comunicación casi inmediato para conocer reacciones personales o grupales frente a iniciativas gubernamentales. También aquí coexisten la posibilidad atractiva y la negativa: la primera es conocer con máxima exactitud y rapidez lo que las personas opinan; la segunda consiste en el riesgo de adecuar las políticas de gobierno a la decisión popular instantánea. Esta tentadora extensión de los alcances del feed back tiene el riesgo de conducir a políticas erráticas o cortoplacistas, si las personas participantes carecen de una visión de conjunto, o no están al tanto de las repercusiones de ciertas medidas en un lapso mayor.

Un último aspecto referido a la tecnología como forma más rápida de participación es la posibilidad de acelerar los cómputos y dar encuestas casi instantáneas en un proceso electoral. Esta posibilidad, que sin duda puede reflejar una información fidedigna de diversos momentos de un proceso participativo, puede generar pro-

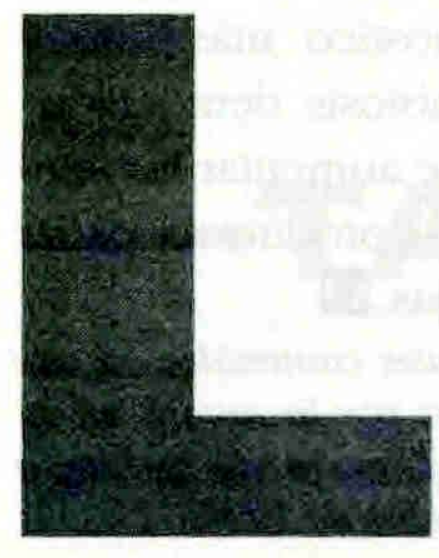

a posibilidad de acelerar los cómputos y dar encuestas casi instantáneas en un proceso electoral puede generar problemas de influencia sesgada en aquellas personas que no hayan ejercido aún su derecho a voto. 
blemas de influencia sesgada en aquellas personas que no hayan ejercido aún su derecho a voto. Ese aspecto ha sido ampliamente controvertido en Chile, donde hay un proyecto de ley que propicia la prohibición de difundir los resultados de las encuestas cinco días antes de las elecciones.

Todas estas posibilidades de la "democracia electrónica" suponen, sin embargo, el acceso de una amplia mayoría a la supercarretera de la información.

\section{CONCLUSION}

La "democracia electrónica", a mediados de los años noventa, sigue entendiéndose más bien como servidora de la transparencia administrativa y del aumento de la disponibilidad de los datos, que como actora real de la bidireccionalidad de los flujos (información desde el público hacia los organismos del Estado y viceversa). Ese tema plantea una serie de interrogantes sobre cambios en las formas de acción del gobierno que aún no están resueltos.

Por otra parte, la amplia difusión de la interconectividad y el acceso de cualquier usuario a entregar información genera problemas en la esfera de la comprobación de la veracidad y confiabilidad de los datos. En esta vertiente, aún muy inexplorada, se enmarca toda la labor del "periodismo electrónico", tanto en lo que se refiere a nuevas formas de reporteo y cruzamiento de datos, como a toda la posibilidad periodística de ofrecer "pautas confiables de navegación" a los usuarios directos.

La efectiva implementación de todos los recursos tecnológicos planteados previamente, requiere de una creciente inversión privada en este campo, una efectiva competencia entre las empresas servidoras de la red, mayor flexibilidad en el uso y en los sistemas de regulación, acceso abierto a todos los usuarios y un servicio lo más universal posible. Estos son los principios básicos de la IMI. A ello se agrega la acción pendiente en capacitación de los usuarios y la obtención de compatibilidad total entre los equipos, cuestión, esta última, en la que se ha avanzado substancialmente con el

\section{BibLIOGRAFía}

BRUNNER, JOSÉ JOAQuin: Procesos de comunicación en una sociedad abierta, Secretaría General de Gobierno, 1995

CASE, TONY: "Los periódicos, los fax, CD ROM y servicios en línea". Editor and Publisher, 1994. CORREA, ENRIQUE: "Comunicaciones: La modernización del sistema en Chile», discurso ante el I Congreso de Comunicación Corporativa, 1995.

COWHEY, PETER F. Y MACKEOWN, MARGARET. "La promesa de un nuevo orden mundial de la información", Servicio Informativo y Cultural de EE.UU., 1995

"Democracia y tecnologia", The Economist, junio de 1995.

"El futuro de la democracia". The Economist, junio de 1995

GoldBerg, SteVEn: Acceso del público a la información de gobierno, U. de Georgetown, 1994.

GORE, AL: "Hacia una infraestructura mundial de la información", discurso del Vicepresidente de los EE.UU. ante la Unión Internacional de las Telecomunicaciones, 1994

MOELLER, PHIL: “La sala de redacción digital», American Journalism Review, 1995.

Moskowitz, RoBert: Globalización de la televisión, Pace Communications, 1995.

NOAM, ELI M: Cuestiones de la política de telecomunicaciones del próximo siglo, Tufts University, 1994. SCHAUER, FREDERICK: La política de comunicaciones y las distracciones de la Primera Enmienda, Universidad de Harvard, 1995

SEPÚlvedA, EduARDO: "Políticos e Internet", El Mercurio, octubre de 1995.

TOFFLER, ALVIN y TOFFLER, HeIDI: Las guerras del futuro, Plaza \& Janés, 1984.

WEBSTER, DAVID: Creación de medios libres, Institute for Contemporary Studies, 1992.

más abierto intercambio de tecnología, que se ha producido entre los gigantes del mundo computacional.

En la perspectiva social, por último, el nuevo mundo de la democracia electrónica plantea dos grandes temas, que sólo es posible dejar enunciados en su formulación inicial:

El primero se refiere a que la democracia habría sufrido algunos embates en la influencia de la comunicación audiovisual. Ello impulsó, en diversos países del mundo, a la elección de dirigentes basados en hábiles campañas publicitarias, que propiciaban una relación puramente emocional con el candidato. La computación y los multimedios, en la medida en que incorporan mayores márgenes de información racional en estos procesos, modifican la relación medio-emoción y moderan políticamente el "efecto aura" de la televisión.

Un segundo aspecto, es el que se refiere a la enorme segmentación del público, con la consiguiente configuración de grupos de interés y compromiso, que exceden ampliamente los tradicionales conformantes de un estado. Las posibilidades de adhesión política se hacen más difíciles o, al menos, muy diferentes de las tradicionales. Esa segmentación se hará también en el aspecto espacial e incluso en las interrelaciones personales, en la medida en que se difunda masivamente el uso de las diferentes formas de telefonía y computación portátiles, así como el de las tablillas electrónicas.

Estos elementos pueden servir de base para iniciar un diagnóstico más fino y matizado sobre la simbiosis democraciatecnología, de modo de aumentar las ventajas comparativas que se producen y paliar sus riesgos y desventajas. Cl

Los conceptos principales contenidos en este articulo fueron expresados por la autora en su participación en una conferencia vía satélite, a través de Worldnet, en octubre de 1995. 